\title{
A importância da alimentação saudável para os portadores de doença autoimune
}

\author{
The importance of healthy food for autoimmune disease patients \\ La importancia de una alimentación saludable para las personas con enfermedades autoinmunes
}

Recebido: 10/09/2021 | Revisado: 16/09/2021 | Aceito: 17/09/2021 | Publicado: 18/09/2021

\author{
Alliandra de Oliveira Farias \\ ORCID: https://orcid.org/0000-0002-0294-1950 \\ Centro Universitário Fametro, Brasil \\ E-mail: alliandrafarias98@gmail.com \\ Antônia Carlos de Melo \\ ORCID: https://orcid.org/0000-0002-9719-1573 \\ Centro Universitário Fametro, Brasil \\ E-mail: anthonyamello@ outlook.com \\ José Carlos de Sales Ferreira \\ ORCID: https://orcid.org/0000-0002-1867-8229 \\ Centro Universitário Fametro, Brasil \\ E-mail: jcarlos.sales@gmail.com
}

\begin{abstract}
Resumo
Introdução: as doenças autoimunes são causadas por uma perda persistente dos mecanismos de controle responsáveis pela manutenção da tolerância aos antígenos próprios. Objetivo Geral: analisar a importância da alimentação equilibrada para os portadores de doença autoimune, bem como os alimentos a serem evitados. Metodologia: Para a realização desse estudo optou-se por uma pesquisa na modalidade de revisão integrativa de literatura. Este é um método de pesquisa apontado como ferramenta de grande relevância no campo da saúde, por proporcionar a busca, a avaliação crítica e a síntese de evidências sobre um tema investigado. Resultados e Discussão: o estado nutricional é extremamente importante no equilíbrio do sistema imunológico, e a composição da dieta assume papel fundamental na manutenção da saúde de todos os indivíduos, inclusive para portadores de doenças autoimunes. Considerações Finais: Através do desenvolvimento deste estudo se pode perceber que a ingesta alimentar é tida como uma importante aliada na redução e prevenção de processos inflamatórios que prejudicam a qualidade de vida, e que podem vir a afetar de forma negativa o quadro clínico do paciente portador de doenças autoimunes.
\end{abstract}

Palavras-chave: Alimentação saudável; Doenças autoimune; Nutrição.

\begin{abstract}
Introduction: autoimmune diseases are caused by a persistent loss of control mechanisms responsible for maintaining tolerance to self antigens. General Objective: to analyze the importance of a balanced diet for people with autoimmune disease, as well as the foods to be avoided. Methodology: To carry out this study, we opted for a research in the modality of integrative literature review. This is a research method pointed out as a tool of great relevance in the field of health, as it provides a search, critical assessment and synthesis of evidence on an investigated topic. Results and Discussion: The nutritional status is extremely important in the balance of the immune system, and the composition of the diet plays a fundamental role in maintaining the health of all individuals, including those with autoimmune diseases. Final Considerations: Through the development of this study, it can be seen that food intake is seen as an important ally in the reduction and prevention of inflammatory processes that impair the quality of life, and that may negatively affect the clinical condition of the carrier patient. of autoimmune diseases.
\end{abstract}

Keywords: Healthy eating; Autoimmune diseases; Nutrition.

\section{Resumen}

Introducción: las enfermedades autoinmunes son causadas por una pérdida persistente de los mecanismos de control encargados de mantener la tolerancia a los autoantígenos. Objetivo general: analizar la importancia de una dieta equilibrada para las personas con enfermedades autoinmunes, así como los alimentos a evitar. Metodología: Para la realización de este estudio se optó por una investigación en la modalidad de revisión integradora de la literatura. Se trata de un método de investigación señalado como una herramienta de gran relevancia en el campo de la salud, ya que proporciona la búsqueda, valoración crítica y síntesis de evidencia sobre un tema investigado. Resultados y Discusión: El estado nutricional es sumamente importante en el equilibrio del sistema inmunológico, y la composición de la dieta juega un papel fundamental en el mantenimiento de la salud de todas las personas, incluidas aquellas con enfermedades autoinmunes. Consideraciones finales: A través del desarrollo de este estudio, se puede apreciar que la ingesta alimentaria se ve como un aliado importante en la reducción y prevención de procesos inflamatorios que 
deterioran la calidad de vida, y que pueden afectar negativamente la condición clínica del paciente portador. . de enfermedades autoinmunes.

Palabras clave: Alimentación saludable; Enfermedades autoinmunes; Nutrición.

\section{Introdução}

As doenças autoimunes são um grupo de mais de 100 doenças relacionadas entre si, que envolvem qualquer órgão ou sistema do nosso organismo. Teorias sugerem que o desenvolvimento de uma doença autoimune requer uma predisposição genética e fatores ambientais, podendo ser infecção por vírus, tabagismo ou menor exposição à luz solar, desencadeando as vias imunológicas que levam, através das inflamações crônicas que não chegam à homeostase e à desorientação do sistema imunológico, atacando o próprio corpo e os órgãos que deveria proteger (Wang et al., 2015).

De acordo com Alwan e Nestle (2015), as doenças autoimunes são causadas por uma perda persistente dos mecanismos de controle responsáveis pela manutenção da tolerância aos antígenos próprios. Para esses autores, uma das características do sistema imune é a sua capacidade de discriminar os antígenos próprios dos não próprios. Essa característica é realizada por linfócitos, capazes de reconhecer e responder contra os antígenos estranhos e não responder contra auto antígenos. Assim, a não expansividade das células do sistema imune contra os antígenos próprios tem sido designada como tolerância imunológica e a perda do controle dos mecanismos que mantêm a tolerância têm sido referidas como autoimunidade.

Mais recentemente, a relação entre a microbiota intestinal e as respostas imunes sistêmicas, incluindo respostas autoimunes, atraiu atenção considerável quanto à patogênese das doenças imunomediadas. Estudos prévios demonstraram de forma conclusiva que a microbiota intestinal é um fator essencial que influencia os componentes celulares e humorais do sistema imunológico intestinal, diminuindo as inflamações crônicas (Horovitz et al., 2013).

Nesse seguimento, sabendo que a relação entre a microbiota intestinal e as respostas imunes sistêmicas, incluindo respostas autoimunes, atraiu atenção considerável quanto à patogênese das doenças imunomediadas, percebe-se a relação de estudos prévios que demonstraram de forma conclusiva que a microbiota intestinal é um fator essencial que influencia os componentes celulares e humorais do sistema imunológico intestinal, diminuindo as inflamações crônicas. E, portanto, o uso de probióticos e prebióticos pode ser uma nova estratégia terapêutica (Villar-García et al., 2017. Miyake et al., 2015).

Diante do exposto, investigar as possíveis interações entre a dieta alimentar e a microbiota intestinal em portadores de doenças autoimune, pode colaborar com o tratamento paliativo ou preventivo da manifestação e até mesmo com a melhora das manifestações agudas ou crônicas da doença.

Desta forma, o objetivo desse estudo é analisar a importância da alimentação equilibrada para os portadores de doença autoimune, bem como os alimentos a serem evitados.

\section{Metodologia}

Para a realização desse estudo optou-se por uma pesquisa na modalidade de revisão integrativa de literatura. A revisão integrativa determina o conhecimento atual sobre uma temática específica, já que é conduzida de modo a identificar, analisar e sintetizar resultados de estudos independentes sobre o mesmo assunto (Silveira et al., 2008).

A Revisão Integrativa é um método de pesquisa apontado como ferramenta de grande relevância no campo da saúde, por proporcionar a busca, a avaliação crítica e a síntese de evidências sobre um tema investigado. Esses aspectos facilitam a identificação dos resultados relevantes, de lacunas que direcionam para o desenvolvimento de futuras pesquisas e auxiliam o profissional a escolher condutas e a tomar decisões, proporcionando um saber crítico (Souza, Silva \& Carvalho, 2010). 
A coleta das informações para a pesquisa bibliográfica se deu por meio da exploração da base de dados da Biblioteca Virtual em Saúde (BVS), Biblioteca Cientifica Eletrônica Virtual (SCIELO) e Literatura Latino-Americana e do Caribe em Ciências da Saúde (LILACS).

A busca na base de dados foi orientada pelos descritores: alimentação saudável, doenças autoimunes, nutrição, e será realizado em todos os índices, buscando captar o maior número de artigos publicados no período proposto que abordem a temática em discussão, visando o desenvolvimento fidedigno desse estudo.

Para uma análise crítica e reflexiva dos estudos incluídos na revisão, foi realizada uma leitura minuciosa e criteriosa destacando os que atingirem os critérios de inclusão e que contemplarem o objetivo proposto, para viabilizar o resultado da pesquisa de forma objetiva.

Foram incluídos os artigos publicados em língua portuguesa nos últimos cinco anos, com texto completo, disponível on-line, com acesso livre. Foram excluídos da amostra os artigos publicados em línguas estrangeiras, os que não apresentarem o texto na integra, artigos que não apresentavam relação direta com o tema, resumos, monografias, dissertações, teses e artigos repetidos.

\section{Resultados e Discussão}

\subsection{A relação entre nutrição e doenças autoimune}

Segundo Machado et al. (2016), as doenças autoimunes são classificadas em sistêmicas ou órgão específicas. Dessa forma, as respostas imunes contra antígenos ou células de vários tecidos produzem doenças sistêmicas, ao passo que a resposta autoimune, contra antígenos de distribuição restrita a tecidos ou grupos celulares, produz doenças órgão específicas.

De maneira geral, a Ingesta alimentar rica em nutrientes influencia no sistema imunológico e se traduz em aumento da imunidade inata associado a uma regulação multifacetada da imunidade adquirida. Em seguimento a isso é apontado que há relação entre a deficiência de vitamina $\mathrm{D}$ e a prevalência de algumas doenças autoimunes como diabetes melito insulinodependente, esclerose múltipla, artrite reumatoide, lúpus sistêmicos e doença inflamatória intestinal (Coan \& Bitencourt, 2019).

$\mathrm{O}$ estado da dieta nutricional refere-se à ingestão de nutrientes da alimentação propriamente dita e também de suplementos, fazendo parte do estado nutricional. Nesse sentido, o estado nutricional é extremamente importante no equilíbrio do sistema imunológico, e a composição da dieta assume papel fundamental na manutenção da saúde de todos os indivíduos, inclusive para portadores de doenças autoimunes como Lúpus Eritematoso Sistêmico (Aureliano, 2018).

Desta forma, o excesso de peso e a obesidade também podem acarretar prejuízos à saúde, conforme sua duração e gravidade, uma vez que também deprimem a resistência, favorecendo infecções. Por provocarem efeitos deletérios na função imunológica, ambos os distúrbios devem ser diagnosticados, com o objetivo de se promover melhor qualidade de saúde às pessoas (Biasebetti, Rodrigues \& Mazur, 2018).

Além disso, de acordo com Freire et al., (2019) há evidências de que fatores dietéticos podem contribuir para a geoepidemiologia de doenças autoimunes. Uma dieta adequada pode, portanto, ser um fator essencial para melhorar o prognóstico de doenças imunológicas, além de ajudar na prevenção de infecç̃oes e na progressão de doenças cardiovasculares.

\subsection{Fatores de risco de déficits nutricionais para portadores de doenças autoimune}

Os hábitos nutricionais de um indivíduo podem causar efeitos cada vez mais impactantes, tanto nos fatores que podem levar ao desenvolvimento de doenças autoimunes, quanto na eficácia de seu tratamento, pois embora o desenvolvimento de doenças autoimune não tenha uma causa propriamente definida, sabe-se que os fatores externos podem causar um grande impacto nessa problemática (Bouillon, 2017). 
Em concordância ao relatado, Freire et al., (2019), relata que o estado nutricional de um indivíduo implica diretamente no equilíbrio e desequilíbrio do seu sistema imunológico. Por este motivo, uma alimentação adequada e equilibrada é imprescindível para a melhora de seu quadro de saúde-doença, podendo auxiliar ainda, na regressão de comorbidades relacionadas e evitar o início de novas infecções.

Bandeira et al., (2016), relata ainda que a ingesta de alimentos com deficiência de vitamina D, ou também conhecido como hipovitaminose D, se estabelece de uma forma sutil, clinicamente apresentando hipocalcemia leve, hiperparatireoidismo reacional, gerando assim perda de osso trabecular e estreitamento de osso cortical. De acordo com o autor, os fatores de risco para a hipovitaminose D são a pouca exposição à luz solar, o envelhecimento da pele e doenças que alterem o metabolismo da vitamina D.

De acordo com Iriart et al., (2019), outro fator de risco para portadores de doenças autoimunes com déficits nutricionais é o desenvolvimento da anemia, a qual seu tratamento e prevenção se dá através de alimentos fontes de ferro e de vitamina $\mathrm{C}$ para o aumento absortivo. Já alimentos como espinafre, café, chá preto ou mate e laticínios junto às refeições principais devem ser evitados, por prejudicarem a biodisponibilidade desse mineral.

O Refluxo gastroesofágico também se caracteriza como um fator de risco para portadores de algumas doenças autoimune, sendo necessário para amenizar esse sintoma, evitar alimentos gordurosos, alimentos à base de cafeína, bebidas alcoólicas, doces e alimentos compostos de purina, por estimular a produção do ácido clorídrico (Antunes et al., 2017).

Segundo Silva et al., (2021) a constipação ou a diarreia também são fatores predominantes nesses pacientes, sendo necessário para evita-los ou controla-los a utilização de fibras alimentares, deve-se evitar ainda o máximo de trabalho digestivo, por meio de uma dieta sem resíduos, é necessário e o consumo de líquidos como água, água de coco, sopas e bebidas isotônicas.

\subsection{Rotina alimentar e doenças autoimune}

Uma rotina alimentar equilibrada e rica em nutrientes, tidos como anti-inflamatória, visam combater e prevenir diversos processos inflamatórios no organismo de pessoas que convivem com doenças autoimunes, como artrite, a qual pode reduzir episódios de crises e surtos, conferindo uma melhor qualidade de vida (Ceccareli, Agmon-Levin \& Perricone, 2016).

De acordo com Zhao et al., (2016), dispor de hábitos nutricionais compostos de alimentos ricos em ômega 3, castanhas e sementes, bem como, frutas cítricas e vermelhas, legumes, iogurtes naturais e outros probióticos, óleo de coco, azeite de oliva e abacate, é imprescindível para uma alimentação equilibrada e anti-inflamatória.

Conforme Marques et al., (2018), é muito importante a ingesta alimentar ou suplementar de vitamina D, pois nos últimos anos o papel fisiológico da vitamina $\mathrm{D}$ tem sido muito estudado mostrando a sua função no metabolismo do cálcio e da formação óssea, bem como, da sua interação com o sistema imunológico. Abrange também uma variedade de tecidos corporais como cérebro, coração, pele, intestino, próstata, mamas e células imunológicas, além de ossos, rins e paratireóides. Para os mesmos autores, a vitamina D tornou-se muito interessante no papel que desempenha, tanto na saúde, como em várias doenças autoimunes, incluindo diabetes mellitus insulinodependente, esclerose múltipla, doença inflamatória intestinal, lúpus sistêmicos e artrite reumatoide.

É relatado ainda por Gerhardt et al., (2016), que o Glutén é um alimento considerado maléfico para os portadores de doenças autoimunes, isso se dá devido a gliadina ser considerada um alérgeno alimentar, que desenvolve reações imune mediadas, podendo desencadear processos inflamatórios severos e também o agravamento ou surgimento de doenças autoimunes.

De acordo com Mehta et al., (2017), assim como o glúten, o leite, mais especificamente a proteína do leite, caseína, também pode vir a ocasionar processos inflamatórios no corpo, e isso pode se dar principalmente em indivíduos com 
intolerância à lactose, que são incapazes de produzir a enzima lactase para a quebra da proteína, levando assim, a uma sensibilização do trato intestinal, causado pelo excesso de lactose não metabolizada, contribuindo para aumentar os sintomas das doenças autoimunes.

\section{Considerações Finais}

Através do desenvolvimento deste estudo se pode perceber que a ingesta alimentar é tida como uma importante aliada na redução e prevenção de processos inflamatórios que prejudicam a qualidade de vida, e que podem vir a afetar de forma negativa o quadro clínico do paciente portador de doenças autoimunes, tendo em vista o valoroso papel que os hábitos alimentares ocasionam no estado de saúde deste.

Ficou evidenciado ainda através deste que as evidências sugerem que a deficiência de vitamina $\mathrm{D}$ pode ter um papel importante na regulação do sistema imunológico e, provavelmente, na prevenção das doenças imunomediadas. No entanto, outros estudos ainda são necessários para determinar os riscos e benefícios da reposição de vitamina D, bem como dos demais componentes nutritivos da dieta desses pacientes.

\section{Referências}

Aguilera, S. L. V. U., França, B. H. S., Moysés, S. T., \& Moysés, S. J. (2014). Iniquidades intermunicipais no acesso e utilização dos serviços de atenção secundária em saúde na região metropolitana de Curitiba. Revista Brasileira de Epidemiologia, 17(3), 654- 667.

Aidar, R. C., et al. (2005). Potencial evocado miogênico vestibular: novas perspectivas diagnosticas em Esclerose múltipla. Revista Brasileira de Otorrinolaringologia, 71(1), 48-54.

Aiello, M., Rampello, A., Granella, F., et al. (2008). Cough efficacy is related to the disability status in patients with multiple sclerosis. Respiration, 76(3), 311-316.

Alonso, M. D., Llorca, J., Martinez-Vazquez, F. et al. (2011). Systemic lupus erythematosus in northwestern Spain: a 20-year epidemiologic study. Medicine, Baltimore, 90, 350-358.

Alwan, W., \& Nestle, F. O. (2015). Pathogenesis and Treatment of Psoriasis: Exploring pathophysiological Pathways for Precision Medicine, Clinical and Experimental Rheumatology, 33(93), 2-6.

Andrade, C. J. N. (2013). Influência dos fatores socioeconômicos e afetivo-emocionais no controle glicêmico de crianças com diabetes mellitus tipo 1. Dissertação (Mestrado) - Programa de Pós-Graduação em Processos Interativos dos Órgãos e Sistemas, Universidade Federal da Bahia. Salvador.

Antunes, B. M., et al. (2017). Imunometabolismo e Exercício Físico: Uma nova fronteira do conhecimento. Motri., Ribeira de Pena, 13(1), 85-98.

Aureliano, W. A. (2018). Trajetórias Terapêuticas Familiares: doenças raras hereditárias como sofrimento de longa duração. Cien Saude Colet, 23(2), 369-380.

Bandeira, F., Griz L., Dreyer, P., Eufrazino, C., Bandeira, C., \& Freese, E. (2016). Vitamin D deficiency: A global perspective. Arq Bras Endocrinol Metabol. $50(4), 640-6$.

Biasebetti, M. B. C., Rodrigues, I. D., \& Mazur, C. E. (2018). Relação do consumo de vitaminas e minerais com o sistema imunitário: uma breve revisão. Visão Acadêmica, Curitiba, 19(1).

Bolon, B. (2012). Cellular and Molecular Mechanisms of Autoimmune Diseases, Journal of Toxicologic Pathology, (40), 216-229.

Bouillon, R. (2017). Comparative analysis of nutritional guidelines for vitamin D. Nature Reviews Endocrinology. 13, 466-479.

Brodin, P., Jojic, V., Gao, T. et al. (2015). Variation in the Human Immune System Is Largely Driven by Non-Heritable Influences. The Cell, 160(1-2), 37-47.

Brown, C. C., \& Noelle, R. J. (2015). Seeing through the dark: New insights into the immune regulatory functions of vitamin A. European Journal Immunology, 45(5), 1287-95.

Buch, E. (2015). Anthropology of Aging and Care. Annual Review of Anthropology 44, 277-293.

Ceccareli, F., Agmon-Levin N., \& Perricone C. (2016). Genetic Factors of Autoimmune Diseases, Journal of Immunology Research.

Choy, E. (2012). Understanding the Dynamics: Pathways Involved in the Pathogenesis of Rheumatoid Arthritis, Rheumatology, 51S (5), 3-11.

Coan F. C., \& Bitencourt R. M. (2019). O uso da vitamina d em doenças autoimunes: revisão sobre o potencial terapêutico. Revista Inova Saúde, Criciúma, 9(1).

De Araújo, A. P. S., Gouvêa, J. A. G., \& Martins, J. (2014). Influência da prática de exercícios físicos sobre os padrões morfofuncionais, função imunológica e qualidade de vida de idosos com aids: estudo de casos. MTP\&Rehabilation Journal, 12, 146-172. 
De Rosa, V., Galgani, M., Santopaolo, M., et al. (2015). Nutritional control of immunity: Balancing the metabolic requirements with an appropriate immune function. Seminars in Immunology, 27(5), 300-9

Domene, S. M. A., Pereira, T. C., \& Arrivillaga, R. K. (2008). Estimated zinc availability in school meals done with standard foods in the city of Campinas (SP), Brazil. Revista de Nutrição, 21(2), 161-167.

Freire, R., Ingano, L., Serena, G., Cetinbas, M., Anselmo, A., Sapone, A., et al. (2019). Human gut derived-organoids provide model to study gluten response and effects of microbiota derived molecules in celiac disease. Nature. 9(7029), 1-15.

Gerhardt T. E., Pinheiro, R., Ruiz, E. N. F., \& Silva Junior, A. G. (2016). Itinerários terapêticos: integralidade no cuidado, avaliação e formação em saúde Rio de Janeiro: CPESC, 13-26.

Horovitz, D. D. G., Ferraz, V. E., Dain, S., \& Marques-De-Farias, A. P. (2013). Genetic services and testing in Brazil. Journal of community genetics. 4(3), $355-375$.

Husby, S., Koletzko, S., Korponay-Szabo, I. R., Kurppa, K., Mearin, M. L., Koninckx, C. R., et al. (2020). European Society for Pediatric Gastroenterology, Hepatology, and Nutrition Guidelines for the Diagnosing Coeliac Disease 2020. J Pediatr Gastroenterol Nutr, 70(1), 141-157.

Iriart, J. A. B., et al. (2019). Da busca pelo diagnóstico às incertezas do tratamento: desafios do cuidado para as doenças genéticas raras no Brasil. Ciênc. saúde coletiva, 24(10), 26.

Liang, Y., Meng, F. Y., Pan, H. F., \& Ye, D. Q. A (2015). LiteratureReview on Patients with Autoimmune Diseases Following Vaccination Against Infections, Human Vaccines and Immunotherapeutics, 11(9), 2274-2280.

Löwy, I. (2018). Tangled diagnoses: Prenatal Testing, Women, and Risk Chicago: The University of Chicago Press.

Luz, G. S., Silva, M. R. S., \& Demontigny, F. (2016). Necessidades prioritárias referidas pelas famílias de pessoas com doenças raras. Texto \& Contexto Enfermagem 25(4), 1-9.

Machado, M. R. C., Gomes Junior, S. C., \& Marinheiro, L. P. F. (2016). Vitamina D e diabetes mellitus, suas epidemias e o envelhecimento. O que há de novo? Reprod clim [Internet]. São Paulo.

Marques, C. D. L, Dantas, A. T., Fragoso, T. S., \& Duarte, Â. L. B. P. (2018). A importância dos níveis de vitamina D nas doenças autoimunes. Rev Bras Reumatol. São Paulo.

Melo, D. G., Germano, C. M. R., Porciúncula, C. G. G., Paiva, I. S., Neri, J. I. C. F, Avó, L. R. S., Demarzo, M. M. P., Galera, M. F. (2017). Qualificação e provimento de médicos no contexto da Política Nacional de Atenção Integral às Pessoas com Doenças Raras no Sistema Único de Saúde (SUS). Interface (Botucatu), 21, 1205-1216.

Mehta, A., et al. (2017). Exploring the patient journey to diagnosis of Gaucher disease from the perspective of 212 patients with Gaucher disease and 16 Gaucher expert physicians. Molecular Genetics and Metabolism, 122(3), 122-129.

Mills, J. R., \& Murray, J. A. (2016). Contemporary celiac disease diagnosis: is a biopsy avoidable? Curr Opin Gastroenterol. 32, 80-85.

Mohammed, G. F., Gomaa, A. H., \& Al-Dhubaibi, M. S. (2015). Highlights in Pathogenesis of Vitiligo, World Journal of Clinical Cases, 3(3), 221-230.

Mohammdibakhsh, R., Sohrabi, R., Salemi, M., Mirghaed, M. T., \& Behzadifar, M. (2017). Celiac disease in Iran: a systematic review and meta-analysis. Electron Physician. 9(3), 3883-3895.

Moreira, M. C. N., Nascimento, M. A. F., Horovitz, D. D. G., Martins, A. J., \& Pinto, M. (2018). Quando ser raro se torna um valor: o ativismo político por direitos das pessoas com doenças raras no Sistema Único de Saúde. Cad Saude Publica.

Muniz, J. G., Sdepanian, V. L., \& Neto, U. F. (2016). Prevalence of genetic susceptibility for celiac disease in blood donors in São Paulo, Brazil. Arq Gastroenterol. 53(4), 267-272.

Parzanese, I., Qehaj, D, Patrinicola, F., Aralica, M., Chirivainternati, M., Stifter, S., et al. (2017). Celiac disease: from pathophysiology to treatment. World J Gastrointest Pathophysiol. 8(2), 27-38.

Rosman, Z., Shoenfeld, V., \& Zandman-Goddard, G. (2013). Biologic therapy for autoimmune diseases: an update. Bio Med Center. 1(88), 1-12. 15.

Rosenkranz, E., Hilgers, R. D., Uciechowski, P., et al. (2015). Zinc enhances the number of regulatory T cells in allergen-stimulated cells from atopic subjects. European Journal of Nutrition, 1-11.

Schwartz, I. V. D., Souza, M. V., Leivas, P. G. C., \& Faccini, L. S. (2014). Clinical genetics and public policies: how should rare diseases be managed? Clinical and Biomedical Research, 34(2), 122-131.

Silva, C. S. F., Cardozo, N. R., Zanatta, R., et al. (2021). Frequency of alleles associated with celiac disease in patients with autoimmune thyroid disease. Rev Nutr, 34 .

Souza, M. T., Silva, M. D., \& Carvalho, R. (2010). Revisão integrativa: o que é e como 15 fazer. Einstein, 8(1), 102-106.

Sorice, A., Guerriero, E., Capone, F., et al. (2014). Ascorbic acid: its role in immune system and chronic inflammation diseases. Mini-Reviews in Medicinal Chemistry, 14(5), 444-52.

Sun, X., Lu, L., Yang, R., Li, Y., Shan, L., \& Wang, Y. (2016). Increased incidence of thyroid disease in patients with celiac disease: a systematic review and meta-analysis. Plos One. 11(12). 
Research, Society and Development, v. 10, n. 12, e233101220452, 2021

(CC BY 4.0) | ISSN 2525-3409 | DOI: http://dx.doi.org/10.33448/rsd-v10i12.20452

Teixeira, T. M., \& Costa, C. L. (2012). Papel da vitamina D no Lúpus Eritematoso Sistemico. Revista de Nutrição, 25(4), 531-538.

Veldhoen, M., \& Ferreira, C. (2015). Influence of nutrient-derived metabolites on lymphocyte immunity. Nature Medicine, 21(7), 709-18.

Zaahur, M., \& Asif, A. R. (2013). Clinical, Cellular and Molecular Biology of autoimmune Disorders - Introduction, Journal of Clinical and Cellular Immunology, 1-2.

Zhao, Z., Zou, J., Zhao, L., Cheng, Y., Cai, H., Li, M., et al. (2016). Celiac disease autoimmunity in patients with autoimmune diabetes and thyroid disease among Chinese population. Plos One. 11(7). 